Geometry $\&$ Topology

Volume 7 (2003) 933-963

Published: 11 December 2003

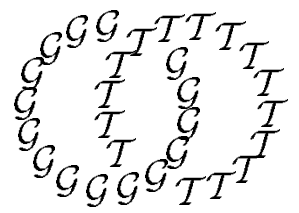

\title{
Combination of convergence groups
}

\author{
FRANÇOIS DAHMANI \\ Forschungsinstitut für Mathematik \\ ETH Zentrum, Rämistrasse, 101 \\ 8092 Zürich, Switzerland. \\ Email: dahmani@math.ethz.ch
}

\begin{abstract}
We state and prove a combination theorem for relatively hyperbolic groups seen as geometrically finite convergence groups. For that, we explain how to contruct a boundary for a group that is an acylindrical amalgamation of relatively hyperbolic groups over a fully quasi-convex subgroup. We apply our result to Sela's theory on limit groups and prove their relative hyperbolicity. We also get a proof of the Howson property for limit groups.
\end{abstract}

AMS Classification numbers Primary: 20F67

Secondary: 20E06

Keywords: Relatively hyperbolic groups, geometrically finite convergence groups, combination theorem, limit groups

Proposed: Benson Farb

Seconded: Jean-Pierre Otal, Walter Neumann
Received: 5 June 2002

Revised: 4 November 2003

(c) Geometry $8 \mathcal{G}$ Topology $\mathcal{P}$ ublications 
The aim of this paper is to explain how to amalgamate geometrically finite convergence groups, or in another formulation, relatively hyperbolic groups, and to deduce the relative hyperbolicity of Sela's limit groups.

A group acts as a convergence group on a compact space $M$ if it acts properly discontinuously on the space of distinct triples of $M$ (see the works of $\mathrm{F}$ Gehring, G Martin, A Beardon, B Maskit, B Bowditch, and P Tukia [12], [1], [6], [30]). The convergence action is uniform if $M$ consists only of conical limit points; the action is geometrically finite (see [1], [5]) if $M$ consists only of conical limit points and of bounded parabolic points. The definition of conical limit points is a dynamical formulation of the so called points of approximation, in the language of Kleinian groups. A point of $M$ is "bounded parabolic" is its stabilizer acts properly discontinuously and cocompactly on its complement in $M$, as it is the case for parabolic points of geometrically finite Kleinian groups acting on their limit sets (see [1], [5]). See Definitions 1.1-1.3 below.

Let $\Gamma$ be a group acting properly discontinuously by isometries on a proper Gromov-hyperbolic space $\Sigma$. Then $\Gamma$ naturally acts by homeomorphisms on the boundary $\partial \Sigma$. If it is a uniform convergence action, $\Gamma$ is hyperbolic in the sense of Gromov, and if the action is geometrically finite, following B Bowditch [8] we say that $\Gamma$ is hyperbolic relative to the family $\mathcal{G}$ of the maximal parabolic subgroups, provided that these subgroups are finitely generated. In such a case, the pair $(\Gamma, \mathcal{G})$ constitutes a relatively hyperbolic group in the sense of Gromov and Bowditch. Moreover, in [8], Bowditch explains that the compact space $\partial \Sigma$ is canonically associated to $(\Gamma, \mathcal{G})$ : it does not depend on the choice of the space $\Sigma$. For this reason, we call it the Bowditch boundary of the relatively hyperbolic group.

The definitions of relative hyperbolicity in [8] (including the one mentionned above) are equivalent to Farb's relative hyperbolicity with the property BCP, defined in [11] (see [29], [8], and the appendix of [10]).

Another theorem of Bowditch [7] states that the uniform convergence groups on perfect compact spaces are exactly the hyperbolic groups acting on their Gromov boundaries. A Yaman [32] proved the relative version of this theorem: geometrically finite convergence groups on perfect compact spaces with finitely generated maximal parabolic subgroups are exactly the relatively hyperbolic groups acting on their Bowditch boundaries (stated below as Theorem 1.5).

We are going to formulate a definition of quasi-convexity (Definition 1.6), generalizing an idea of Bowditch described in [6]. A subgroup $H$ of a geometrically finite convergence group on a compact space $M$ is fully quasi-convex if it is geometrically finite on its limit set $\Lambda H \subset M$, and if only finitely many translates of 
$\Lambda H$ can intersect non trivially together. We also use the notion of acylindrical amalgamation, formulated by Sela [23], which means that there is a number $k$ such that the stabilizer of any segment of length $k$ in the Serre tree, is finite.

Theorem 0.1 (Combination theorem)

(1) Let $\Gamma$ be the fundamental group of an acylindrical finite graph of relatively hyperbolic groups, whose edge groups are fully quasi-convex subgroups of the adjacent vertices groups. Let $\mathcal{G}$ be the family of the images of the maximal parabolic subgroups of the vertices groups, and their conjugates in $\Gamma$. Then, $(\Gamma, \mathcal{G})$ is a relatively hyperbolic group.

(2) Let $G$ be a group which is hyperbolic relative to a family of subgroups $\mathcal{G}$, and let $P$ be a group in $\mathcal{G}$. Let $A$ be a finitely generated group in which $P$ embeds as a subgroup. Then, $\Gamma=A *_{P} G$ is hyperbolic relative to the family $(\mathcal{H} \cup \mathcal{A})$, where $\mathcal{H}$ is the set of the conjugates of the images of elements of $\mathcal{G}$ not conjugated to $P$ in $G$, and where $\mathcal{A}$ is the set of the conjugates of $A$ in $\Gamma$.

(3) Let $G_{1}$ and $G_{2}$ be relatively hyperbolic groups, and let $P$ be a maximal parabolic subgroup of $G_{1}$, which is isomorphic to a parabolic (not necessarly maximal) subgroup of $G_{2}$. Let $\Gamma=G_{1} *_{P} G_{2}$. Then $\Gamma$ is hyperbolic relative to the family of the conjugates of the maximal parabolic subgroups of $G_{1}$, except $P$, and of the conjugates of the maximal parabolic subgroups of $G_{2}$.

$\left(3^{\prime}\right)$ Let $G$ be a relatively hyperbolic group and let $P$ be a maximal parabolic subgroup of $G$ isomorphic to a subgroup of another parabolic subgroup $P^{\prime}$ not conjugated to $P$. Let $\Gamma=G *_{P}$ according to the two images. Then $\Gamma$ is hyperbolic relative to the family of the conjugates of the maximal parabolic subgroups of $G$, except $P$ (but including the parabolic group $P^{\prime}$ ).

Up to our knowledge, the assumption of finite generation of the maximal parabolic subgroups is useful for a proof of the equivalence of different definitions of relative hyperbolicity. For the present work, it is not essential, and without major change, one can state a combination theorem for groups acting as geometrically finite convergence groups on metrisable compact spaces in general.

A first example of application of the main theorem is already known as a consequence of Bestvina and Feighn Combination Theorem [3], [4], where there are no parabolic group: acylindrical amalgamations of hyperbolic groups over quasi-convex subgroups satisfy the first case of the theorem (see Proposition 1.11). Another important example is the amalgamation of relatively hyperbolic 
groups over a parabolic subgroup, which is stated as the third and fourth case. They are in fact consequences of the two first cases.

Instead of choosing the point of view of Bestvina and Feighn [3], [4], and constructing a hyperbolic space on which the group acts in an adequate way (see also the works of R Gitik, O Kharlampovich, A Myasnikov, and I Kapovich, [13], [21], [18]), we adopt a dynamical point of view: from the actions of the vertex groups on their Bowditch's boundaries, we construct a metrizable compact space on which $\Gamma$ acts naturally, and we check (in section 3 ) that this action is of convergence and geometrically finite. At the end of the third part, we prove the Theorem 0.1 using Bowditch-Yaman's Theorem 1.5.

In other words, we construct directly the boundary of the group $\Gamma$. This is done by gluing together the boundaries of the stabilizers of vertices in the BassSerre tree, along the limit sets of the stabilizers of the edges. This does not give a compact space, but the boundary of the Bass-Serre tree itself naturally compactifies it. This construction is explained in detail in section 2 .

Thus, we have a good description of the boundary of the amalgamation. In particular:

Theorem 0.2 (Dimension of the boundary)

Under the hypothesis of Theorem 0.1, let $\partial \Gamma$ be the boundary of the relatively hyperbolic group $\Gamma$. If the topological dimensions of the boundaries of the vertex groups (resp. of the edge groups) are smaller than $r$ (resp. than $s$ ), then $\operatorname{dim}(\partial \Gamma) \leq \operatorname{Max}\{r, s+1\}$.

The application we have in mind is the study of Sela's limit groups, or equivalently $\omega$-residually free groups [24], [22]. In part 4, we answer the first question of Sela's list of problems [25].

Theorem 0.3 Limit groups are hyperbolic relative to their maximal abelian non-cyclic subgroups.

This allows us to get some corollaries.

Corollary 0.4 Every limit group satisfies the Howson property: the intersection of two finitely generated subgroups of a limit group is finitely generated.

Corollary 0.5 Every limit group admits a $\mathcal{Z}$-structure in the sense of Bestvina $([2],[9])$. 
The first corollary was previously proved by I Kapovich in [19], for hyperbolic limit groups (see also [20]).

I am grateful to T Delzant, for his interest, and advices, and to Z Sela who suggested the problem about limit groups to me. I also want to thank B Bowditch, I Kapovich, G Swarup, and F Paulin for their comments and questions. Finally I am deeply grateful to the referee for his/her remarks.

\section{Geometrically finite convergence groups, and rel- ative hyperbolicity}

\subsection{Definitions}

We recall the definitions of [1], [6] and [30].

Definition 1.1 (Convergence groups)

A group $\Gamma$ acting on a metrizable compact space $M$ is a convergence group on $M$ if it acts properly discontinuously on the space of distinct triples of $M$.

If the compact space $M$ has more than two points, this is equivalent to say that the action is of convergence if, for any sequence $\left(\gamma_{n}\right)_{n \in \mathbb{N}}$ of elements of $\Gamma$, there exists two points $\xi$ and $\zeta$ in $M$, and a subsequence $\left(\gamma_{\phi(n)}\right)_{n \in \mathbb{N}}$, such that for any compact subspace $K \subset M \backslash\{\xi\}$, the sequence $\left(\gamma_{\phi(n)} K\right)_{n \in \mathbb{N}}$, uniformly converges to $\zeta$.

Definition 1.2 (Conical limit point, bounded parabolic point)

Let $\Gamma$ be a convergence group on a metrizable compact space $M$. A point $\xi \in M$ is a conical limit point if there exists a sequence in $\Gamma,\left(\gamma_{n}\right)_{n \in \mathbb{N}}$, and two points $\zeta \neq \eta$, in $M$, such that $\gamma_{n} \xi \rightarrow \zeta$ and $\gamma_{n} \xi^{\prime} \rightarrow \eta$ for all $\xi^{\prime} \neq \xi$.

A subgroup $G$ of $\Gamma$ is parabolic if it is infinite, fixes a point $\xi$, and contains no loxodromic element (a loxodromic element is an element of infinite order fixing exactly two points in the boundary). In this case, the fixed point of $G$ is unique and is referred to as a parabolic point. Such a point $\xi \in M$ is bounded parabolic if its stabilizer $\operatorname{Stab}(\xi)$ acts properly discontinuously co-compactly on $M \backslash\{\xi\}$.

Note that the stabilizer of a parabolic point is a maximal parabolic subgroup of $\Gamma$. 
Definition 1.3 (Geometrically finite groups)

A convergence group on a compact space $M$ is geometrically finite if $M$ consists only of conical limit points and bounded parabolic points.

Here is a geometrical counterpart (see [14], [8]).

Definition 1.4 (Relatively hyperbolic groups)

We say that a group $\Gamma$ is hyperbolic relative to a family of finitely generated subgroups $\mathcal{G}$, if it acts properly discontinuously by isometries, on a proper hyperbolic space $\Sigma$, such that the induced action on $\partial \Sigma$ is of convergence, geometrically finite, and such that the maximal parabolic subgroups are exactly the elements of $\mathcal{G}$.

In this situation we also say that the pair $(\Gamma, \mathcal{G})$ is a relatively hyperbolic group.

The boundary of $\Sigma$ is canonical in this case (see [8]); we call it the boundary of the relatively hyperbolic group $(\Gamma, \mathcal{G})$, or the Bowditch boundary, and we write it $\partial \Gamma$.

As recalled in the introduction, one has:

Theorem 1.5 (Yaman [32], Bowditch [7] for groups without parabolic subgroups)

Let $\Gamma$ be a geometrically finite convergence group on a perfect metrizable compact space $M$, and let $\mathcal{G}$ be the family of its maximal parabolic subgroups. Assume that each element of $\mathcal{G}$ is finitely generated. Assume that there are only finitely many orbits of bounded parabolic points. Then $(\Gamma, \mathcal{G})$ is relatively hyperbolic, and $M$ is equivariantly homeomorphic to $\partial \Gamma$.

In fact, by a result of Tukia ([31], Theorem 1B), the assumption of finiteness of the set of orbits of parabolic points can be omitted. With this dictionary between geometrically finite convergence groups, and relatively hyperbolic groups, we will sometimes say that a group $\Gamma$ is relatively hyperbolic with Bowditch boundary $\partial \Gamma$, when we mean that the pair $(\Gamma, \mathcal{G})$ is relatively hyperbolic, where $\mathcal{G}$ is the family of maximal parabolic subgroups in the action on $\partial \Gamma$. 


\section{$1.2 \quad$ Fully quasi-convex subgroups}

Let $\Gamma$ be a convergence group on $M$. According to [6], the limit set $\Lambda H$ of an infinite non virtually cyclic subgroup $H$, is the unique minimal non-empty closed $H$-invariant subset of $M$. The limit set of a virtually cyclic subgroup of $\Gamma$ is the set of its fixed points in $M$, and the limit set of a finite group is empty. We will use this for relatively hyperbolic groups acting on their Bowditch boundaries.

Definition 1.6 (Quasi-convex and fully quasi-convex subgroups)

Let $\Gamma$ be a relatively hyperbolic group, with Bowditch boundary $\partial \Gamma$, and let $H$ be a group acting as a geometrically finite convergence group on a compact space $\partial H$. We assume that $H$ embeds in $\Gamma$ as a subgroup. We say that $H$ is quasi-convex in $\Gamma$ if its limit set $\Lambda H \subset \partial \Gamma$ is equivariantly homeomorphic to $\partial H$.

It is fully quasi-convex if it is quasi-convex and if, for any infinite sequence $\left(\gamma_{n}\right)_{n \in \mathbb{N}}$ all in distinct left cosets of $H$, the intersection $\bigcap_{n}\left(\gamma_{n} \Lambda H\right)$ is empty.

Remark (i) If $H$ is a subgroup of $\Gamma$, and if $\Gamma$ acts as a convergence group on a compact space $M$, every conical limit point for $H$ acting on $\Lambda H \subset M$, is a conical limit point for $H$ acting in $M$, and therefore, even for $\Gamma$ acting on $M$. Therefore it is not a parabolic point (see the result of Tukia, described in [6] Prop.3.2, see also [31]), and each parabolic point for $H$ in $\Lambda H$ is a parabolic point for $\Gamma$ in $M$, and its maximal parabolic subgroup in $H$ is exactly the intersection of its maximal parabolic subgroup in $\Gamma$ with $H$.

Remark (ii) if $H$ is a quasiconvex subgroup of a relatively hyperbolic group $\Gamma$, and if its maximal parabolic subgroups are finitely generated, then it is hyperbolic relative to these maximal parabolic subgroups (by Theorem 1.5), hence it is finitely generated. In particular, it is always the case when the parabolic subgroups of $\Gamma$ are finitely generated abelian groups.

Remark (iii) If $H \leq G \leq \Gamma$ are three relatively hyperbolic groups, such that $G$ is fully quasi-convex in $\Gamma$, and $H$ is fully quasi-convex in $G$, then $H$ is fully quasi-convex in $\Gamma$. Indeed, the limit set of $H$ in $\Gamma$ is the image of the limit set of $H$ in $G$ by the equivariant inclusion map $\partial(G) \hookrightarrow \partial(\Gamma)$.

Lemma 1.7 ('Full' intersection with parabolic subgroups)

Let $\Gamma$ be a relatively hyperbolic group with boundary $\partial \Gamma$, and $H$ be a fully quasi-convex subgroup. Let $P$ be a parabolic subgroup of $\Gamma$. Then $P \cap H$ is either finite, or of finite index in $P$. 
Let $p \in \partial \Gamma$ the parabolic point fixed by $P$. Assume $P \cap H$ is not finite, so that $p \in \Lambda H$. Then $p$ is in every translate of $\Lambda H$ by an element of $P$. The second point of Definition 1.6 shows that there are finitely many such translates: $P \cap H$ is of finite index in $P$.

Proposition 1.8 Let $(\Gamma, \mathcal{G})$ be a relatively hyperbolic group, and $\partial \Gamma$ its Bowditch boundary. Let $H$ be a quasi-convex subgroup of $\Gamma$, and $\Lambda H$ be its limit set in $\partial \Gamma$. Let $\left(\gamma_{n}\right)_{n \in \mathbb{N}}$ be a sequence of elements of $\Gamma$ all in distinct left cosets of $H$. Then there is a subsequence $\left(\gamma_{\sigma(n)}\right)$ such that $\gamma_{\sigma(n)} \Lambda H$ uniformly converges to a point.

Unfortunately I do not know any purely dynamical proof of this proposition, that would only involve the geometrically finite action on the boundary.

There is a proper hyperbolic geodesic space $X$, with boundary $\partial \Gamma$, on which $\Gamma$ acts properly discontinuously by isometries. We assume that $\Lambda H$ contains two points $\xi_{1}$ and $\xi_{2}$, otherwise the result is a consequence of the compactness of $\partial \Gamma$. Let $B(\Lambda H)$ be the union of all the bi-infinite geodesic between points of $\Lambda H$ in $X$, and $p$ be a point in it. Note that $B(\Lambda H)$ is quasi-convex in $X$, and that $H$ acts on it properly discontinuously by isometries. We prove that the boundary $\partial(B(\Lambda H))$ of $B(\Lambda H)$ is precisely $\Lambda H$. Indeed, if $p_{n}$ is a sequence of points in $B(\Lambda H)$ going to infinity, there are bi-infinite geodesics $\left(\xi_{n}, \zeta_{n}\right)$ containing each $p_{i}$, with $\xi_{n}$ and $\zeta_{n}$ in $\Lambda H$. Let us extract a subsequence such that $\left(\xi_{n}\right)_{n}$ converges to a point $\xi \in \partial(\Gamma)$, and $\zeta_{n} \rightarrow \zeta \in \partial(\Gamma)$. As $\Lambda H$ is closed, $\xi$ and $\zeta$ are in it, and the sequence $\left(p_{n}\right)_{n}$ must converge to one of these two points (or both if they are equal).

By our definition of quasi-convexity, $H$ acts on $\partial(B \Lambda H)=\Lambda H$ as a geometrically finite convergence group.

To prove the proposition, it is enough to prove that a subsequence of the sequence $\operatorname{dist}\left(\gamma_{n}^{-1} p, B(\Lambda H)\right)$ tends to infinity. Indeed, by quasi-convexity of $B(\Lambda H)$ in $X$, for all $\xi$ and $\zeta$ in $\Lambda H$, the Gromov products $\left(\gamma_{n} \xi \cdot \gamma_{n} \zeta\right)_{p}$ are greater than $\operatorname{dist}\left(\gamma_{n}^{-1} p, B(\Lambda H)\right)-K$, where $K$ depends only on $\delta$ and on the quasi-convexity constant of $B(\Lambda H)$. Thus, we now want to prove that a subsequence of $\operatorname{dist}\left(\gamma_{n}^{-1} p, B(\Lambda H)\right)$ tends to infinity.

For all $n$, let $h_{n} \in H$ be such that $\operatorname{dist}\left(h_{n} p, \gamma_{n}^{-1} p\right)$ is minimal among the distances $\operatorname{dist}\left(h p, \gamma_{n}^{-1} p\right), h \in H$. We prove the lemma:

Lemma 1.9 The sequence $\left(\operatorname{dist}\left(h_{n} p, \gamma_{n}^{-1} p\right)\right)_{n}$ tends to infinity. 
Indeed, if a subsequence was bounded by a number $N$, then for infinitely many indexes, the point $h_{n}^{-1} \gamma_{n}^{-1} p$ is in the ball of $X$ of center $p$ and of radius $N$. Therefore, there exists $n$ and $m \neq n$ such that $h_{n}^{-1} \gamma_{n}^{-1}=h_{m}^{-1} \gamma_{m}^{-1}$, which contradicts our hypothesis that all the $\gamma_{n}$ are in distinct left cosets of $H$.

Let us resume the proof of Proposition 1.8. For all $n$, let now $q_{n}$ be a point in $B(\Lambda H)$ such that $\operatorname{dist}\left(\gamma_{n}^{-1} p, B(\Lambda H)\right)=\operatorname{dist}\left(\gamma_{n}^{-1} p, q_{n}\right)$. By the triangular inequality, $\operatorname{dist}\left(q_{n}, \gamma_{n}^{-1} p\right) \geq \operatorname{dist}\left(h_{n} p, \gamma_{n}^{-1} p\right)-\operatorname{dist}\left(h_{n} p, q_{n}\right)$. If $\left(\operatorname{dist}\left(h_{n} p, q_{n}\right)\right)_{n}$ does not tend to infinity, then a subsequence of $\left(\operatorname{dist}\left(q_{n}, \gamma_{n}^{-1} p\right)\right)_{n}$ tends to infinity and we are done. Assume now that $\left(\operatorname{dist}\left(h_{n} p, q_{n}\right)\right)_{n}$ tends to infinity. After translation by $h_{n}^{-1}$, the sequence $\left(\operatorname{dist}\left(p, h_{n}^{-1} q_{n}\right)\right)_{n}$ tends to infinity. Recall an usual result (Proposition 6.7 in [8]): given a $\Gamma$-invariant system of horofunctions $\left(\rho_{\xi}\right)_{\xi \in \Pi}$, for the set $\Pi$ of bounded parabolic points in $\partial \Gamma$, for all $t$, there exists only finitely many horofunctions $\rho_{\xi_{1}} \ldots \rho_{\xi_{k}}$ such that $\rho_{\xi_{i}}(p) \geq t$. As there are finitely many orbits of bounded parabolic points in $\Lambda H$, it is possible to choose $t$ such that for every $\xi \in \Pi \cap \Lambda H$, there exists $h \in H$ such that $\rho_{\xi}(h p) \geq t+1$. The group $H$, as a geometrically finite group, acts co-compactly in the complement of a system of horoballs in $B(\Lambda H)$ (Proposition 6.13 in [8]). By definition of the elements $h_{n}$, for all $h \in H$, one has $\operatorname{dist}\left(h p, h_{n}^{-1} q_{n}\right) \geq \operatorname{dist}\left(p, h_{n}^{-1} q_{n}\right)$, and the latter tends to infinity. Therefore the sequence $h_{n}^{-1} q_{n}$ leaves the complement of any system of horoballs. In other words, for all $M>0$, there exists $n_{0}$ such that for all $n \geq n_{0}$, there is $i \in\{1, \ldots, k\}$ such that $\rho_{\xi_{i}}\left(h_{n}^{-1} q_{n}\right) \geq M$.

Therefore, one can extract a subsequence such that for some horofunction $\rho$ associated to a bounded parabolic point in $\Lambda H, \rho\left(h_{n}^{-1} q_{n}\right)$ tends to infinity. If $\operatorname{dist}\left(h_{n}^{-1} q_{n}, h_{n}^{-1} \gamma_{n}^{-1} p\right)$ remains bounded, then $\rho\left(h_{n}^{-1} \gamma_{n}^{-1} p\right)$ tends to infinity, which is in contradiction with Lemma 6.6 of [8], because $h_{n}^{-1} \gamma_{n}^{-1} p$ is in the $\Gamma$-orbit of $p$. Therefore a subsequence of $\operatorname{dist}\left(h_{n}^{-1} q_{n}, h_{n}^{-1} \gamma_{n}^{-1} p\right)$ tends to infinity, and after translation by $h_{n}$, this gives the result: a subsequence of $\operatorname{dist}\left(B(\Lambda H), \gamma_{n}^{-1} p\right)$ tends to infinity.

The following statement appears in [15] and also in [26], for hyperbolic groups. Note that this is no longer true for (non fully) quasi-convex subgroups.

Proposition 1.10 (Intersection of fully quasi-convex subgroups)

Let $\Gamma$ be a relatively hyperbolic group with boundary $\partial \Gamma$. If $H_{1}$ and $H_{2}$ are fully quasi-convex subgroups of $\Gamma$, then $H_{1} \cap H_{2}$ is fully quasi-convex, moreover $\Lambda\left(H_{1} \cap H_{2}\right)=\Lambda H_{1} \cap \Lambda H_{2}$.

As, for $i=1$ and $2, H_{i}$ is a convergence group on $\Lambda H_{i}$, and as any sequence of distinct translates of $\Lambda H_{i}$ has empty intersection, the same is true for $H_{1} \cap H_{2}$ on $\Lambda H_{1} \cap \Lambda H_{2}$. 
Let $p \in\left(\Lambda H_{1} \cap \Lambda H_{2}\right)$ a parabolic point for $\Gamma$, and $P<\Gamma$ its stabilizer. For $i=1$ and 2 , the group $H_{i} \cap P$ is maximal parabolic in $H_{i}$, hence infinite. By Lemma 1.7, they are both of finite index in $P$, and therefore so is their intersection. Hence $p$ is a bounded parabolic point for $H_{1} \cap H_{2}$ in $\left(\Lambda H_{1} \cap \Lambda H_{2}\right)$.

Let $\xi \in\left(\Lambda H_{1} \cap \Lambda H_{2}\right)$ be a conical limit point for $\Gamma$. Then, by the first remark after the definition of quasi-convexity, it is a conical limit point for each of the $H_{i}$.

According to the definition of conical limit points, let $\left(\gamma_{n}\right)_{n \in \mathbb{N}}$ be a sequence of elements in $\Gamma$ such that there exists $\zeta$ and $\eta$ two distinct points in $\partial \Gamma$, with $\gamma_{n} \xi \rightarrow \zeta$, and $\gamma_{n} \xi^{\prime} \rightarrow \eta$ for all other $\xi^{\prime}$. There exists a subsequence of $\left(\gamma_{n}\right)_{n \in \mathbb{N}}$ staying in a same left coset of $H_{1}$ : if not, the fact that two sequences $\left(\gamma_{n} \xi\right)_{n \in \mathbb{N}}$ and $\left(\gamma_{n} \xi^{\prime}\right)_{n \in \mathbb{N}}$, for $\xi^{\prime} \in \Lambda H_{1} \backslash\{\xi\}$ converge to two different points contradicts the Proposition 1.8. By the same argument, there exists a subsequence of the previous subsequence that remains in a same left coset of $H_{1}$, and in a same left coset of $H_{2}$. Therefore it stays in a same left coset of $H_{1} \cap H_{2}$; we can assume that we chose the sequence $\left(\gamma_{n}\right)_{n \in \mathbb{N}}$ such that there exists $\gamma \in \Gamma$ and $\left(h_{n}\right)_{n \in \mathbb{N}}$ a sequence of elements of $H_{1} \cap H_{2}$, such that $\forall n, \gamma_{n}=\gamma h_{n}$.

Therefore $h_{n} \xi \rightarrow \gamma^{-1} \zeta$, and $h_{n} \xi^{\prime} \rightarrow \gamma^{-1} \eta$ for all other $\xi^{\prime}$. This means that $\xi \in \Lambda\left(H_{1} \cap H_{2}\right)$ is a conical limit point for the action of $\left(H_{1} \cap H_{2}\right)$. This ends the proof of Proposition 1.10.

We emphasize the case of hyperbolic groups, studied by Bowditch in [6].

Proposition 1.11 (Case of hyperbolic groups)

In a hyperbolic group, a proper subgroup is quasi-convex in the sense of quasiconvex subsets of a Cayley graph, if and only if it is fully quasi-convex.

B Bowditch proved in [6] that a subgroup $H$ of a hyperbolic group $\Gamma$ is quasiconvex if and only if it is hyperbolic with limit set equivariantly homeomorphic to $\partial H$. It remains only to see that, if $H$ is quasi-convex in the classical sense, then the intersection of infinitely many distinct translates $\bigcap_{n \in \mathbb{N}}\left(\gamma_{n} \partial H\right)$ is empty, and we prove it by contradiction. Let us choose $\xi$ in $\bigcap_{n \in \mathbb{N}}\left(\gamma_{n} \partial H\right)$. Then, there is $L>0$ depending only on the quasi-convexity constant of $H$ in $\Gamma$, and there is, in each coset $\gamma_{n} H$, an L-quasi-geodesic ray $r_{n}(t)$ tending to $\xi$. As they converge to the same point in the boundary of a hyperbolic space, there is a constant $D$ such that for all $i$ and $j$ we have: $\exists t_{i, j} \forall t>t_{i, j}, \exists t^{\prime}, \operatorname{dist}\left(r_{i}(t), r_{j}\left(t^{\prime}\right)\right)<D$. Let $N$ be a number larger than the number of vertices in the a of radius $D$ in the Cayley graph of $\Gamma$, and consider a point $r_{1}(T)$ with $T$ bigger than any $t_{i, j}$, for $i, j \leq N$. Then each ray $r_{i}$, 
$i \leq N$, has to pass through the ball of radius $D$ centered in $r_{1}(T)$. By a pigeon hole argument, we see that two of them pass through the same vertex, but they were supposed to be in disjoint cosets.

Our point of view in Definition 1.6 is a generalization of the definitions in [6], given for hyperbolic groups.

\section{Boundary of an acylindrical graph of groups}

Let $\Gamma$ be as the first or the second part of Theorem 0.1. We will say that we are in Case (1) (resp. in Case (2)) if $\Gamma$ satisfies the first (resp. the second) assumption of Theorem 0.1. However, we will need this distinction only for the proof of Proposition 2.2.

Let $T$ be the Bass-Serre tree of the splitting, and $\tau$, a subtree of with $T$ which is a fundamental domain. We assume that the action of $\Gamma$ on $T$ is $k$-acylindrical for some $k \in \mathbb{N}$ (from Sela [23]): the stabilizer of any segment of length $k$ is finite.

We fix some notation: if $v$ is a vertex of $T, \Gamma_{v}$ is its stabilizer in $\Gamma$. Similarly, for an edge $e$, we write $\Gamma_{e}$ for its stabilizer. For a vertex $v, \Gamma_{v}$ is relatively hyperbolic. This is by assumption in Case (1), and in Case (2), if $\Gamma_{v}$ is conjugated to $A$, we consider that it is hyperbolic relative to itself; in this case the space $\Sigma$ of Definition 1.4 is just an horoball, and its Bowditch boundary is a single point. For the existence of such a hyperbolic horoball, notice that the second definition of Bowditch [8] indicates that the group $A * A$ is hyperbolic relative to the conjugates of both factors. Indeed we do not need to know the existence of such an horoball, but only that $A$ acts as a geometrically finite convergence group on a single point, which is trivial.

\subsection{Definition of $M$ as a set}

\section{Contribution of the vertices of $T$}

Let $\mathcal{V}(\tau)$ be the set of vertices of $\tau$. For a vertex $v \in \mathcal{V}(\tau)$, the group $\Gamma_{v}$ is by assumption a relatively hyperbolic group and we denote by $\partial\left(\Gamma_{v}\right)$ a compact space homeomorphic to its Bowditch boundary. Thus, $\Gamma_{v}$ is a geometrically finite convergence group on $\partial\left(\Gamma_{v}\right)$.

We set $\Omega$ to be $\Gamma \times\left(\bigsqcup_{v \in \mathcal{V}(\tau)} \partial\left(\Gamma_{v}\right)\right)$ divided by the natural relation

$$
\left(\gamma_{1}, x_{1}\right)=\left(\gamma_{2}, x_{2}\right) \text { if } \exists v \in \mathcal{V}(\tau), x_{i} \in \partial\left(\Gamma_{v}\right), \gamma_{2}^{-1} \gamma_{1} \in \Gamma_{v}, \gamma_{2}^{-1} \gamma_{1} x_{1}=x_{2}
$$


In particular, for each $v \in \tau$, the compact space $\partial \Gamma_{v}$ naturally embeds in $\Omega$ as the image of $\{1\} \times \partial \Gamma_{v}$. We identify it with its image. The group $\Gamma$ naturally acts on the left on $\Omega$. The compact space $\gamma \partial\left(\Gamma_{v}\right)$ is called the boundary of the vertex stabilizer $\Gamma_{\gamma v}$.

\section{Contribution of the edges of $T$}

Each edge will allow us to glue together boundaries of vertex stabilizers along the limit sets of the stabilizer of the edge. We explain precisely how.

For an edge $e=\left(v_{1}, v_{2}\right)$ in $\tau$, the group $\Gamma_{e}$ embeds as a quasi-convex subgroup in both $\Gamma_{v_{i}}, i=1,2$. Thus, by definition of quasi-convexity, these embedings define equivariant maps $\Lambda_{\left(e, v_{i}\right)}: \partial\left(\Gamma_{e}\right) \hookrightarrow \partial\left(\Gamma_{v_{i}}\right)$, where $\partial\left(\Gamma_{e}\right)$ is the Bowditch boundary of the relatively hyperbolic group $\Gamma_{e}$. Similar maps are defined by translation, for edges in $T \backslash \tau$.

The equivalence relation $\sim$ on $\Omega$ is the transitive closure of the following: for $v$ and $v^{\prime}$ are vertices of $T$, the points $\xi \in \partial\left(\Gamma_{v}\right)$ and $\xi^{\prime} \in \partial\left(\Gamma_{v^{\prime}}\right)$ are equivalent in $\Omega$ if there is an edge $e$ between $v$ and $v^{\prime}$, and a point $x \in \partial\left(\Gamma_{e}\right)$ satisfying simultaneously $\xi=\Lambda_{(e, v)}(x)$ and $\xi^{\prime}=\Lambda_{\left(e, v^{\prime}\right)}(x)$.

Lemma 2.1 Let $\pi$ be the projection corresponding to the quotient: $\pi: \Omega \rightarrow$ $\Omega / \sim$. For all vertex $v$, the restriction of $\pi$ on $\partial\left(\Gamma_{v}\right)$ is injective.

Let $\xi$ and $\xi^{\prime}$ be two points of $\Omega$, both of them being in the boundary of a vertex stabilizer $\partial\left(\Gamma_{v}\right)$. If they are equivalent for the relation above, then there is a sequence of consecutive edges $e_{1}=\left(v, v_{1}\right), e_{2}=\left(v_{1}, v_{2}\right) \ldots e_{n}=\left(v_{n-1}, v\right)$, the first one starting at $v_{0}=v$ and the last one ending at $v_{n}=v$, and a sequence of points $\xi_{i} \in \partial\left(\Gamma_{v_{i}}\right)$, for $i \leq n-1$, such that, for all $i$, there exists $x_{i} \in \partial\left(\Gamma_{e_{i}}\right)$, satisfying $\xi_{i}=\Lambda_{\left(e_{i}, v_{i-1}\right)}\left(x_{i}\right)$ and $\xi_{i+1}=\Lambda_{\left(e_{i}, v_{i}\right)}\left(x_{i}\right)$. As $T$ is a tree, it contains no simple loop, and there exists an index $i$ such that $v_{i-1}=v_{i+1}$. As, for all $j$, the maps $\Lambda_{\left(e_{j}, v_{j}\right)}$ are injective, the points $\xi_{i-1}$ and $\xi_{i+1}$ are the same in $\left.\partial\left(\Gamma_{(} v_{i-1}\right)\right)$, and inductively, we see that $\xi$ and $\xi^{\prime}$ are the same point. This proves the claim.

Note that the group $\Gamma$ acts on the left on $\Omega / \sim$. Let $\partial T$ be the (visual) boundary of the tree $T$ : it is the space of the rays in $T$ starting at a given base point; let us recall that for its topology, a sequence of rays $\left(\rho_{n}\right)$ converges to a given ray $\rho$, if $\rho_{n}$ and $\rho$ share arbitrarily large prefixes, for $n$ large enough. We define $M$ as a set:

$$
M=\partial T \sqcup(\Omega / \sim) .
$$


As before, let $\pi$ be the projection corresponding to the quotient: $\pi: \Omega \rightarrow \Omega / \sim$. For a given edge $e$ with vertices $v_{1}$ and $v_{2}$, the two maps $\pi \circ \Lambda_{\left(e, v_{i}\right)}: \partial\left(\Gamma_{e}\right) \rightarrow$ $\Omega / \sim(i=1,2)$, are two equal homeomorphisms on their common image. We identify this image with the Bowditch boundary of $\Gamma_{e}, \partial\left(\Gamma_{e}\right)$, and we call this compact space, the boundary of the edge stabilizer $\Gamma_{e}$.

\subsection{Domains}

Let $\mathcal{V}(T)$ be the set of vertices of $T$. We still denote by $\pi$ the projection: $\pi: \Omega \rightarrow \Omega / \sim$. Let $\xi \in \Omega / \sim$. We define the domain of $\xi$, to be $D(\xi)=\{v \in$ $\left.\mathcal{V}(T) \mid \xi \in \pi\left(\partial\left(\Gamma_{v}\right)\right)\right\}$. As we want uniform notations for all points in $M$, we say that the domain of a point $\xi \in \partial T$ is $\{\xi\}$ itself.

Proposition 2.2 (Domains are bounded)

For all $\xi \in \Omega / \sim, D(\xi)$ is convex in $T$, its diameter is bounded by the acylindricity constant, and the intersection of two distinct domains is finite. The quotient of $D(\xi)$ by the stabilizer of $\xi$ is finite.

Remark In Case (1), we will even prove that domains are finite, but this is false in Case (2).

The equivalence $\sim$ in $\Omega$ is the transitive closure of a relation involving points in boundaries of adjacent vertices, hence domains are convex.

End of the proof in Case (2) As $P$ is a maximal parabolic subgroup of $G$, its limit set is a single point: $\partial(P)$ is one point belonging to the boundary of only one stabilizer of an edge adjacent to the vertex $v_{G}$ stabilized by $G$. Hence, the domain of $\xi=\partial\left(\Gamma_{v_{A}}\right)$ is $\left\{v_{A}\right\} \cup \operatorname{Link}\left(v_{A}\right)$, that is $v_{A}$ with all its neighbours, whereas the domain of a point $\zeta$ which is not a translate of $\partial\left(\Gamma_{v_{A}}\right)$, is only one single vertex.

Domains have therefore diameter bounded by 2 , and any two of them intersect only on one point. For the last assertion, note that $A$ stabilizes the point $\partial\left(\Gamma_{v_{A}}\right)$, and acts transitively on the edges adjacent to $v_{A}$. This proves the lemma in Case (2).

In Case (1), we need a lemma:

Lemma 2.3 In Case (1), let $\xi \in \Omega / \sim$. The stabilizer of any finite subtree of $D(\xi)$ is infinite. 
If a subtree, whose vertices are $\left\{v_{1}, \ldots, v_{n}\right\}$, is in $D(\xi)$, then there exists a group $H$ embedded in each of the vertex stabilizers $\Gamma_{v_{i}}$ as a fully quasi-convex subgroup, with $\xi$ in its limit set.

The first assertion is clearly a consequence of the second one, we will prove the latter by induction.

If $n=1, H$ is the vertex stabilizer. For larger $n$, re-index the vertices so that $v_{n}$ is a final leaf of the subtree $\left\{v_{1}, \ldots, v_{n}\right\}$, with unique neighbor $v_{n-1}$. Let $e$ be the edge $\left\{v_{n-1}, v_{n}\right\}$. The induction gives $H_{n-1}$, a subgroup of the stabilizers of each $v_{i}, i \leq n-1$, and with $\xi \in \partial H_{n-1}$. As $\xi \in \partial\left(\Gamma_{v_{n}}\right)$, it is in $\partial\left(\Gamma_{e}\right)$, and we have $\xi \in \partial H_{n-1} \cap \partial\left(\Gamma_{e}\right)$. By Proposition $1.10, H_{n-1} \cap \Gamma_{e}$ is a fully quasi-convex subgroup of $\Gamma_{v_{n-1}}$, and therefore, it is a a fully quasi-convex subgroup of $\Gamma_{e}$, and of $H_{n-1}$. Therefore, (see Remark (iii)), it is a fully quasi-convex subgroup of $\Gamma_{v_{n}}$, and of each of the $\Gamma_{i}$, for $i \leq(n-1)$, with $\xi$ in its limit set. It is then adequate for $H$; this proves the claim, and Lemma 2.3 .

End of the proof of Prop. 2.2 in Case (1) By Lemma 2.3, each segment in $D(\xi)$ has an infinite stabilizer, hence by $k$-acylindricity, $\operatorname{Diam}(D(\xi)) \leq$ $k$. Domains are bounded, and they are locally finite because of the second requirement of Definition 1.6, therefore they are finite. The other assertions are now obvious.

\subsection{Definition of neighborhoods in $M$}

We will describe $\left(W_{n}(\xi)\right)_{n \in \mathbb{N}, \xi \in M}$, a family of subsets of $M$, and prove that it generates an topology (Theorem 2.10) which is suitable for our purpose.

For a vertex $v$, and an open subset $U$ of $\partial\left(\Gamma_{v}\right)$, let $T_{v, U}$ be the subtree whose vertices $w$ are such that $[v, w]$ starts by an edge $e$ with $\partial\left(\Gamma_{e}\right) \cap U \neq \emptyset$.

For each vertex $v$ in $T$, let us choose $\mathcal{U}(v)$, a countable basis of open neighborhoods of $\partial\left(\Gamma_{v}\right)$, seen as the Bowditch boundary of $\Gamma_{v}$. Without loss of generality, we can assume that for all $v$, the collection of open subsets $\mathcal{U}(v)$ contains $\partial\left(\Gamma_{v}\right)$ itself.

Let $\xi$ be in $\Omega / \sim$, and $D(\xi)=\left\{v_{1}, \ldots, v_{n}, \ldots\right\}=\left(v_{i}\right)_{i \in I}$. Here, the set $I$ is a subset of $\mathbb{N}$. For each $i \in I$, let $U_{i} \subset \partial\left(\Gamma_{v_{i}}\right)$ be an element of $\mathcal{U}\left(v_{i}\right)$, containing $\xi$, such that for all but finitely many indices $i \in I, U_{i}=\partial\left(\Gamma_{v_{i}}\right)$.

The set $W_{\left(U_{i}\right)_{i \in I}}(\xi)$ is the disjoint union of three subsets $W_{\left(U_{i}\right)_{i \in I}}(\xi)=A \cup B \cup C$ : 
- $A=\bigcap_{i \in I} \partial\left(T_{v_{i}, U_{i}}\right)$,

- $B=\left\{\zeta \in(\Omega / \sim) \backslash\left(\bigcup_{i \in I} \partial\left(\Gamma_{v_{i}}\right)\right) \mid D(\zeta) \subset \bigcap_{i \in I} T_{v_{i}, U_{i}}\right\}$

- $C=\left\{\zeta \in \bigcup_{j \in I} \partial\left(\Gamma_{v_{j}}\right) \mid \zeta \in \bigcap_{m \in I \mid \zeta \in \partial\left(\Gamma_{v_{m}}\right)} U_{m}\right\}$.

Remark The set of elements of $\Omega / \sim$ is not countable, nevertheless, the set of different possible domains is countable. Indeed a domain is a finite subset of vertices of $T$ or the star of a vertex of $T$, and this makes only countably many possibilities. The set $W_{\left(U_{i}\right)_{i \in I}}(\xi)$ is completely defined by the data of the domain of $\xi$, the data of a finite subset $J$ of $I$, and the data of an element of $\mathcal{U}\left(v_{j}\right)$ for each index $j \in J$. Therefore, there are only countably many different sets $W_{\left(U_{i}\right)_{i \in I}}(\xi)$, for $\xi \in \Omega / \sim$, and $U_{i} \in \mathcal{U}\left(v_{i}\right), v_{i} \in D(\xi)$. For each $\xi$ we choose an arbitrary order and denote them $W_{m}(\xi)$.

Let us choose $v_{0}$ a base point in $T$. For $\xi \in \partial T$, we define the subtree $T_{m}(\xi)$ : it consists of the vertices $w$ such that $\left[v_{0}, w\right] \cap\left[v_{0}, \xi\right)$ has length bigger than $m$. We set $W_{m}(\xi)=\left\{\zeta \in M \mid D(\zeta) \subset T_{m}(\xi)\right\}$. Up to a shift in the indexes, this does not depend on $v_{0}$, for $m$ large enough.

Lemma 2.4 (Avoiding an edge)

Let $\xi$ be a point in $M$, and $e$ an edge in $T$ with at least one vertex not in $D(\xi)$. Then, there exists an integer $n$ such that $W_{n}(\xi) \cap \partial\left(\Gamma_{e}\right)=\emptyset$.

If $\xi$ is in $\partial T$ the claim is obvious. If $\xi \in \Omega / \sim$, as $T$ is a tree, there is a unique segment from the convex $D(\xi)$ to $e$. Let $v$ be the vertex of $D(\xi)$ where this path starts, and $e_{0}$ be its first edge. It is enough to find a neighborhood of $\xi$ in $\partial\left(\Gamma_{v}\right)$ that misses $\partial\left(\Gamma_{e_{0}}\right)$. As one vertex of $e_{0}$ is not in $D(\xi), \xi$ is not in $\partial\left(\Gamma_{e_{0}}\right)$, which is compact. Hence such a separating neighborhood exists.

\subsection{Topology of $M$}

In the following, we consider the smallest topology $\mathcal{T}$ on $M$ such that the family of sets $\left\{W_{n}(\xi) ; n \in \mathbb{N}, \xi \in M\right\}$, with the notations above, are open subsets of $M$.

Lemma 2.5 The topology $\mathcal{T}$ is Hausdorff.

Let $\xi$ and $\zeta$ two points in $M$. If the subtrees $D(\xi)$ and $D(\zeta)$ are disjoint, there is an edge $e$ that separates them in $T$, and Lemma 2.4 gives two neighborhoods of the points that do not intersect. Even if $D(\xi) \cap D(\zeta)$ is non-empty, it is 
nonetheless finite (Proposition 2.2). In each of its vertex $v_{i}$, we can choose disjoint neighborhoods $U_{i}$ and $V_{i}$ for the two points. This gives rise to sets $W_{n}(\xi)$ and $W_{m}(\zeta)$ which are separated.

\section{Lemma 2.6 (Filtration)}

For every $\xi \in M$, every integer $n$, and every $\zeta \in W_{n}(\xi)$, there exists $m$ such that $W_{m}(\zeta) \subset W_{n}(\xi)$.

If $D(\zeta)$ and $D(\xi)$ are disjoint, again, Lemma 2.4 gives a neighborhood of $\zeta$, $W_{m}(\zeta)$ that do not meet $\partial\left(\Gamma_{e}\right)$, whereas $\partial\left(\Gamma_{e}\right) \subset W_{n}(\xi)$, because $\zeta \in W_{n}(\xi)$. By definition of our family of neighborhoods, $W_{m}(\zeta) \subset W_{n}(\xi)$.

If the domains of $\xi$ and $\zeta$ have a non-trivial intersection, either the two points are equal (and there is nothing to prove), or the intersection is finite (Prop. 2.2). Let $\left(v_{i}\right)_{i \in I}=D(\xi)$, let $\left(U_{i}\right)_{i \in I}$ be such that $W_{n}(\xi)=W_{\left(U_{i}\right)_{i}}(\xi)$, and let $J \subset I$ be such that $D(\xi) \cap D(\zeta)=\left(v_{j}\right)_{j \in J}$. In this case, we can choose, for all $j \in J$, a neighbourhood of $\zeta$ in $\partial\left(\Gamma_{j}\right), U_{j}^{\prime} \subset U_{j}$ such that $U_{j}^{\prime}$ do not meet the boundary of the stabilizer of an edge $\left(v_{j}, v_{i}\right)$ for any $i \in I \subset J$; this gives $W_{m}(\zeta) \subset W_{n}(\xi)$.

Corollary 2.7 The family $\left\{W_{n}(\xi)\right\}_{n \in \mathbb{N}, \xi \in M}$ is a fundamental system of open neighborhoods of $M$ for the topology $\mathcal{T}$.

It is enough to show that the intersection of two such sets is equal to the union of some other ones. Let $W_{n_{1}}\left(\xi_{1}\right)$ and $W_{n_{2}}\left(\xi_{2}\right)$ be in the family. Let $\zeta$ be in their intersection. Lemma 2.6 gives $W_{\left(U_{j}\right)_{j}}(\zeta) \subset W_{n_{1}}\left(\xi_{1}\right)$ and $W_{\left(V_{j}\right)_{j}}(\zeta) \subset$ $W_{n_{2}}\left(\xi_{2}\right)$. As $W_{\left(U_{j}\right)_{j}}(\zeta) \cap W_{\left(V_{j}\right)_{j}}(\zeta)=W_{\left(U_{j} \cap V_{j}\right)_{j}}(\zeta)$, we get an integer $m_{\zeta}$ such that $W_{m_{\zeta}}(\zeta)$ is included in both $W_{n_{i}}\left(\xi_{i}\right)$. Therefore, $W_{n_{1}}\left(\xi_{1}\right) \cap W_{n_{2}}\left(\xi_{2}\right)=$ $\bigcup_{\zeta \in W_{n_{1}}\left(\xi_{1}\right) \cap W_{n_{2}}\left(\xi_{2}\right)} W_{m_{\zeta}}(\zeta)$.

Corollary 2.8 Recall that $\pi$ be the projection corresponding to the quotient: $\pi: \Omega \rightarrow \Omega / \sim$. For all vertex $v$, the restriction of $\pi$ on $\partial\left(\Gamma_{v}\right)$ is continuous.

Let $\xi$ be in $\partial\left(\Gamma_{v}\right)$, and let $\left(\xi_{n}\right)_{n}$ be a sequence of elements of $\partial\left(\Gamma_{v}\right)$ converging to $\xi$ for the topology of $\partial\left(\Gamma_{v}\right)$. Let $\left(U^{n}\right)_{n}$ be a system of neighbourhoods of $\xi$ in $\partial\left(\Gamma_{v}\right)$, such that for all $n$, for all $n^{\prime} \geq n, \xi_{n^{\prime}} \in U^{n}$. Let $D(\pi(\xi))=$ $\left\{v, v_{2}, \ldots\right\}$ in $T$, and consider $W_{m}=W_{\left(U_{i}(m)\right)}(\pi(\xi))$, such that $U_{1}(m) \subset U^{n}$. By definition, $W_{\left(U_{i}(m)\right)}(\pi(\xi)) \cap \pi\left(\partial\left(\Gamma_{v}\right)\right)$ is the image by $\pi$ of an open subset of $U_{1}(m)$ containing $\xi$. Therefore, by property of fundamrental systems of neighbourhoods, $\pi\left(\xi_{n}\right)$ converges to $\pi(\xi)$. Therefore $\pi$ is continuous. 
From now, we identify $\xi$ and $\pi(\xi)$ in such situation.

Lemma 2.9 The topology $\mathcal{T}$ is regular, that is, for all $\xi$, for all $m$, there exists $n$ such that $\overline{U_{n}(\xi)} \subset U_{m}(\xi)$.

In the case of $\xi \in \partial T$, the closure of $W_{n}(\xi)$ is contained in $W_{n}^{\prime}(\xi)=\{\zeta \in$ $\left.M \mid D(\zeta) \cap T_{n}(\xi) \neq \emptyset\right\}$ (compare with the definition of $W_{n}(\xi)$ ). As, by Proposition 2.2, domains have uniformly bounded diameters, we see that for arbitrary $m$, if $n$ is large enough, $\overline{W_{n}(\xi)} \subset W_{m}(\xi)$.

In the case of $\xi \in \Omega / \sim, \overline{W_{\left(U_{i}\right)_{i}}(\xi)} \backslash W_{\left(U_{i}\right)_{i}}(\xi)$ contains only points in the boundaries of vertices of $D(\xi)$, and those are in the closure of the $U_{i}$ (which is non-empty only for finitely many $i$ ), and in the boundary (not in $U_{i}$ ) of edges meeting $U_{i} \backslash\{\xi\}$. Therefore, given $V_{i} \subset \partial\left(\Gamma_{v_{i}}\right)$, with strict inclusion only for finitely many indices, if we choose the $U_{i}$ small enough to miss the boundary of every edge non contained in $V_{i}$, except the ones meeting $\xi$ itself, we have $\overline{W_{\left(U_{i}\right)_{i}}(\xi)} \subset W_{\left(V_{i}\right)_{i}}(\xi)$.

Theorem 2.10 Let $\Gamma$ be as in Theorem 0.1. With the notations above, $\left\{W_{n}(\xi) ; n \in \mathbb{N}, \xi \in M\right\}$ is a base of a topology that makes $M$ a perfect metrizable compact space, with the following convergence criterion: $\left(\xi_{n} \rightarrow \xi\right) \Longleftrightarrow$ $\left(\forall n \exists m_{0} \forall m>m_{0}, \xi_{m} \in W_{n}(\xi)\right)$.

The topology is, by construction, second countable, separable. As it is also Hausdorff (Lemma 2.5) and regular (Lemma 2.9), it is metrizable. The convergence criterion is an immediate consequence of Corollary 2.7. Let us prove that it is sequentially compact. Let $\left(\xi_{n}\right)_{n \in \mathbb{N}}$ be a sequence in $M$, we want to extract a converging subsequence. Let us choose $v$ a vertex in $T$, and for every $n, v_{n} \in D\left(\xi_{n}\right)$ minimizing the distance to $v$ (if $\xi_{n} \in \partial T$, then $v_{n}=\xi_{n}$ ). There are two possibilities (up to extracting a subsequence): either the Gromov products $\left(v_{n} \cdot v_{m}\right)_{v}$ remain bounded, or they go to infinity. In the second case, the sequence $\left(v_{n}\right)_{n}$ converges to a point in $\partial T$, and by our convergence criterion, we see that $\left(\xi_{n}\right)_{n}$ converges to this point (seen in $\partial T \subset M$ ). In the first case, after extraction of a subsequence, one can assume that the Gromov products $\left(v_{n} \cdot v_{m}\right)_{v}$ are constant equal to a number $N$. Let $g_{n}$ be a geodesic segment or a geodesic ray between $v$ and $v_{n}$. there is a segment $g=\left[v, v^{\prime}\right]$ of length $N$, which is contained in every $g_{n}$, and for all distinct $n$ and $m, g_{n}$ and $g_{m}$ do not have a prefix longer than $g$.

Either there is a subsequence so that $g_{n_{k}}=g$ for all $n_{k}$, and as $\partial \Gamma_{v^{\prime}}$ is compact, this gives the result, or there is a subsequence such that every $g_{n_{k}}$ is strictly 
longer than $g$. Let $e_{n_{k}}$ be the edge of $g_{n_{k}}$ following $v^{\prime}$. All the $e_{n_{k}}$ are distinct, therefore, by Proposition 1.8, one can extract another subsequence such that the sequence of the boundaries of their stabilizers converge to a single point of $\partial \Gamma_{v^{\prime}}$. The convergence criterion indicates that the subsequence of $\left(\xi_{n_{k}}\right)_{n}$ converges to this point.

Therefore, $M$ is sequentially compact and metrisable, hence it is compact. It is perfect since $\partial T$ has no isolated point, and accumulates everywhere.

Theorem 2.11 (Topological dimension of $M$ ) [Theorem 0.2]

$\operatorname{dim}(M) \leq \max _{v, e}\left\{\operatorname{dim}\left(\partial\left(\Gamma_{v}\right)\right), \operatorname{dim}\left(\partial\left(\Gamma_{e}\right)\right)+1\right\}$.

It is enough to show that every point has arbitrarily small neighborhoods whose boundaries have topological dimension at most $(n-1)$ (see the book [16], where this property is set as a definition).

If $\xi \in \partial T$, the closure of $W_{n}(\xi)$ is contained in $W_{n}^{\prime}(\xi)=\left\{\zeta \in M \mid D(\zeta) \cap T_{n}(\xi) \neq\right.$ $\emptyset\}$ (compare with the definition of $W_{n}(\xi)$ ). The boundary of $W_{n}(\xi)$ is therefore a compact subspace of the boundary of the stabilizer of the unique edge that has one and only one vertex in $T_{n}(\xi)$; the boundary of $W_{n}(\xi)$ has dimension at $\operatorname{most}_{\max }\left\{\operatorname{dim}\left(\partial\left(\Gamma_{e}\right)\right)\right\}$.

If $\xi \in \Omega / \sim, \overline{W_{\left(U_{i}\right)_{i}}(\xi)} \backslash W_{\left(U_{i}\right)_{i}}(\xi)$ contains only points in the boundaries of vertices of $D(\xi)$, and those are in the closure of the $U_{i}$ (which is non-empty only for finitely many $i$ ), and in the boundaries (not in $U_{i}$ ) of stabilizers of edges that meet $U_{i} \backslash\{\xi\}$. Hence, the boundary of a neighborhood $W_{n}(\xi)$ is the union of boundaries of neighborhoods of $\xi$ in $\partial\left(\Gamma_{v_{i}}\right)$ and of a compact subspace of the boundary of countably many stabilizers of edges. As the dimension of a countable union of compact spaces of dimension at most $n$ is of dimension at most $n$ (Theorem III.2 in [16]), its dimension is therefore at most $\max _{v, e}\left\{\operatorname{dim}\left(\partial\left(\Gamma_{v}\right)\right)-1, \operatorname{dim}\left(\partial\left(\Gamma_{e}\right)\right)\right\}$. This proves the claim.

\section{Dynamic of $\Gamma$ on $M$}

We assume the same hypothesis as for Theorem 2.10. We first prove two lemmas, and then we prove the different assertions of Theorem 3.7.

Lemma 3.1 (Large translations)

Let $\left(\gamma_{n}\right)_{n \in \mathbb{N}}$ be a sequence in $\Gamma$. Assume that, for some (hence any) vertex $v_{0} \in T$, $\operatorname{dist}\left(v_{0}, \gamma_{n} v_{0}\right) \rightarrow \infty$. Then, there is a subsequence $\left(\gamma_{\sigma(n)}\right)_{n \in \mathbb{N}}$, there 
is a point $\zeta \in M$, and a point $\zeta^{\prime} \in \partial T$, such that for all compact subspace $K \subset\left(M \backslash\left\{\zeta^{\prime}\right\}\right)$, one has $\gamma_{\sigma(n)} K \rightarrow \zeta$ uniformly.

Let $\xi_{0}$ be in $\partial\left(\Gamma_{v_{0}}\right)$. Using the sequential compactness of $M$, we choose a subsequence $\left(\gamma_{\sigma(n)}\right)_{n \in \mathbb{N}}$ such that $\left(\gamma_{\sigma(n)} \xi_{0}\right)_{n}$ converges to a point $\zeta$ in $M$; we still have $\operatorname{dist}\left(v_{0}, \gamma_{\sigma(n)} v_{0}\right) \rightarrow \infty$.

Let $v_{1}$ be another vertex in $T$. The lengths of the segments $\left[\gamma_{n} v_{0}, \gamma_{n} v_{1}\right]$ are all equal to the length of $\left[v_{0}, v_{1}\right]$, therefore, for all $m$, there is $n_{m}$ such that for all $n>n_{m}$, the segments $\left[v_{0}, \gamma_{\sigma(n)} v_{0}\right]$ and $\left[v_{0}, \gamma_{\sigma(n)} v_{1}\right]$ have a common prefix of length more than $m$.

Let $\zeta_{1}, \zeta_{2}$ in $\partial T$. The center of the triangle $\left(v_{0}, \zeta_{1}, \zeta_{2}\right)$ is a vertex $v$ in $T$. Therefore, for all $m \geq 0$, the segments $\left[v_{0}, \gamma_{\sigma(n)} v_{0}\right]$ and $\left[v_{0}, \gamma_{\sigma(n)} v\right]$ coincide on a subsegment of length more than $m$, for sufficiently large integers $n$. This means that for at least one of the $\zeta_{i}$, the ray $\left[v_{0}, \gamma_{\sigma(n)} \zeta_{i}\right)$ has a common prefix with $\left[v_{0}, \gamma_{\sigma(n)} v_{0}\right]$ of length at least $m$. By convergence criterion, $\left(\gamma_{\sigma(n)} \zeta_{i}\right)$ converges to $\zeta$. Therefore there exits $\zeta^{\prime}$ in $\partial \Gamma$, such that any other point $\zeta " \in\left(\partial T \backslash\left\{\zeta^{\prime}\right\}\right)$, satisfies $\gamma_{\sigma(n)} \zeta " \rightarrow \zeta$.

Let $K$ be a compact subspace of $\left(M \backslash\left\{\zeta^{\prime}\right\}\right)$. There exists a vertex $v_{0}$, a point $\xi \in \partial T$, and a neighborhood $W_{m}(\xi)$ (see the definition in the section above, where $v_{0}$ is the base point) of $\xi$ containing $K$, not containing $\zeta^{\prime}$. Let $v$ be on the ray $\left[v_{0}, \xi\right)$, at distance $m$ from $v_{0}$. Then for all points $\xi^{\prime}$ in $W_{m}(\xi)$ the ray $\left[v_{0}, \xi^{\prime}\right)$ has the prefix $\left[v_{0}, v\right]$. As the sequence $\left(\gamma_{\sigma(n)} \partial \Gamma_{v}\right)_{n \in \mathbb{N}}$ uniformly converges to $\zeta$, the sequence $\left(\gamma_{\sigma(n)} W_{m}(\xi)\right)_{n \in \mathbb{N}}$ uniformly converges to this point. Therefore, the convergence is uniform on $K$.

Lemma 3.2 (Small translation)

Let $\left(\gamma_{n}\right)_{n \in \mathbb{N}}$ be a sequence of distinct elements of $\Gamma$, and assume that for some (hence any) vertex $v_{0}$, the sequence $\left(\gamma_{n} v_{0}\right)_{n}$ is bounded in $T$. Then there exists a subsequence $\left(\gamma_{\sigma(n)}\right)_{n \in \mathbb{N}}$, a vertex $v$, a point $\zeta \in \partial\left(\Gamma_{v}\right)$, and another point $\zeta^{\prime} \in \Omega / \sim$, such that, for all compact subspace $K$ of $M \backslash\left\{\zeta^{\prime}\right\}$, one has $\gamma_{\sigma(n)} K \rightarrow \zeta$ uniformly.

We distinguish two cases. First, we assume that for some vertex $v$, and for some element $\gamma \in \Gamma$, there exists a subsequence such that $\gamma_{n}=h_{n} \gamma$, with $h_{n} \in \Gamma_{v}$ for all $n$. In such a case, we can extract again a subsequence (but, without loss of generality, we still denote it by $\left.\left(\gamma_{n}\right)_{n}\right)$ such that there exists a point $\zeta^{\prime} \in \partial\left(\Gamma_{\gamma^{-1} v}\right)$ and a point $\zeta \in \partial\left(\Gamma_{v}\right)$, such that for every compact subspace $K_{\gamma^{-1} v} \subset \partial\left(\Gamma_{\gamma^{-1} v}\right) \backslash\left\{\zeta^{\prime}\right\}$, our subsequence of $\gamma_{n} K_{\gamma^{-1} v}$ converges to $\zeta$ uniformly. 
Assume that $\zeta^{\prime}$ is not a parabolic point for $\Gamma_{v}$ in $\partial\left(\Gamma_{v}\right)$. For any vertex $w$ in $D\left(\gamma \zeta^{\prime}\right)$, let $e$ be the first edge of the segment $[v, w]$. The boundary of its stabilizer contains $\zeta^{\prime}$. The elements $h_{n}$ are all, except finitely many, in the same left coset of $\operatorname{Stab}(e)$, otherwise, as $h_{n} \gamma \zeta^{\prime}$ and $h_{n} \xi$ go to different points, for all $\xi \neq \gamma \zeta^{\prime}$ in $\partial\left(\Gamma_{e}\right) \backslash\left\{\zeta^{\prime}\right\}$ (which is non empty since $\zeta^{\prime}$ is not parabolic), we get a contradiction with Proposition 1.8. Therefore, we can extract a subsequence (but, without loss of generality, we still denote it by $\left.\left(\gamma_{n}\right)_{n}\right)$ such that, for each vertex $\gamma^{-1} w \in D\left(\zeta^{\prime}\right)$, for each compact subspace $K_{\gamma^{-1} w}$ of $\partial\left(\Gamma_{\gamma^{-1} w}\right)$, not containing $\zeta^{\prime}$, the sequence $\gamma_{n} K_{\gamma^{-1} w}$ converges to $\zeta$ uniformly. Assume now that $\zeta^{\prime}$ is a parabolic point for $\Gamma_{v}$ in $\partial\left(\Gamma_{v}\right)$. Then $h_{n}\left(\gamma \zeta^{\prime}\right)$ do converge to $\zeta$, otherwise, $\zeta^{\prime}$ would be a conical limit point. Therefore, for all vertex $\gamma^{-1} w \in D\left(\zeta^{\prime}\right) \backslash\left\{\gamma^{-1} v\right\}$, the sequence $\gamma_{n} \partial\left(\Gamma_{\gamma^{-1} w}\right)$ converges to $\zeta$ uniformly.

Therefore, if $v^{\prime}$ is a vertex not in the domain of $\zeta^{\prime}$, the path from $\gamma^{-1} v$ to $v^{\prime}$ contains an edge such that the boundary of its stabilizer is a compact space $K_{\gamma^{-1} w}$ satisfying: $\gamma_{n} K_{\gamma^{-1} w} \rightarrow \zeta^{\prime}$ uniformly. Let $K$ be a compact subspace of $M \backslash\left\{\zeta^{\prime}\right\}$. For each $v_{i} \in D\left(\zeta^{\prime}\right)$, there exists a compact space $K_{i} \subset \partial\left(\Gamma_{v_{i}}\right) \backslash\left\{\zeta^{\prime}\right\}$, $K \cap \partial\left(\Gamma_{v_{i}}\right) \subset K_{i}$ such that for all other point $\xi$ of $K$, the unique ray in $T$ from $D\left(\zeta^{\prime}\right)$ that converges to $\xi$ contains an edge such that the boundary of its stabilizer is contained in some $K_{i}$. Therefore, $\gamma_{n} K \rightarrow \zeta^{\prime}$ uniformly.

We turn now to the second case, where such a subsequence does not exists. Nevertheless, after extraction, we can assume that the distance $\operatorname{dist}\left(v_{0}, \gamma_{n} v_{0}\right)$ is constant. Let $v$ be the vertex such that there exists a subsequence $\left(\gamma_{\sigma(n)}\right)_{n \in \mathbb{N}}$ with the property that some segments $\left[v_{0}, \gamma_{\sigma(n)} v_{0}\right]$ have a common prefix $\left[v_{0}, v\right]$, and the edges $e_{\sigma(n)} \subset\left[v_{0}, \gamma_{\sigma(n)} v_{0}\right]$ located just after $v$, are all distinct. By Proposition 1.8, one can extract a subsequence $\left(e_{\sigma^{\prime}(n)}\right)_{n}$ such that the boundaries of the stabilizers of these edges converge to some point $\zeta \in \partial\left(\Gamma_{v}\right)$. By our convergence criterion, $\gamma_{\sigma^{\prime}(n)} \partial\left(\Gamma_{v_{0}}\right)$ uniformly converges to $\zeta$.

Let $\xi$ be a point in $\partial T$. We claim that $v$ is not in the ray $\left[\gamma_{\sigma^{\prime}(n)} v_{0}, \gamma_{\sigma^{\prime}(n)} \xi\right)$, for $n$ sufficiently large. If it was, there would be a subsequence satisfying: $\gamma_{\sigma^{\prime}(n)}^{-1} v$ is constant on a vertex $w$ of the ray $\left[v_{0}, \xi\right)$, that is, $\gamma_{\sigma^{\prime}(n)}^{-1}=h_{n} \gamma$, where $h_{n} \in \Gamma_{w}$. Therefore, $\gamma_{\sigma^{\prime}(n)} w$ equals $v$ for all $n$. In other words, for all $n$ there exists $h_{n}$ in $\Gamma_{w}$ such that $\gamma_{\sigma^{\prime}(n)}=h_{n} \gamma_{\sigma^{\prime}(0)}$. This contradicts our assumption that we are not in the first case, and this proves the claim.

If $d=\operatorname{dist}\left(\gamma_{\sigma^{\prime}(n)} v_{0}, v\right)$ (which is constant by assumption), we choose the neighborhood of $\xi$ defined by $W_{d+1}(\xi)$ (here $v_{0}$ is the base point). Then, for each point in $\gamma_{\sigma^{\prime}(n)} W_{d+1}(\xi)$, the unique path in $T$ from $v_{0}$ to this point contains $e_{n}$. Therefore, $\gamma_{\sigma^{\prime}(n)} W_{d+1}(\xi)$, uniformly converges to $\zeta$. 
Let $\xi$ be now a point in the boundary of the stabilizer of a vertex $v^{\prime}$. Again, for the same reason, the vertex $v$ is not in $\left[\gamma_{\sigma^{\prime}(n)} v_{0}, \gamma_{\sigma(n)}^{\prime} v^{\prime}\right]$ for $n$ large enough. Therefore the unique path from $v$ to $\gamma_{\sigma^{\prime}(n)} v^{\prime}$ contains the edge $e_{\sigma^{\prime}(n)}$. If $\gamma_{\sigma^{\prime}(n)} \xi$ is not in $\partial\left(\Gamma_{e_{\sigma^{\prime}(n)}}\right)$, for all $n$ sufficiently large, then there exists a neighborhood $N$ of $\xi$ such that the convergence $\gamma_{\sigma^{\prime}(n)} N \rightarrow \zeta$ is uniform. If $\gamma_{\sigma^{\prime}(n)} \xi$ is in $\partial\left(\Gamma_{e_{\sigma^{\prime}(n)}}\right)$, then there exists another vertex $v_{n}^{\prime \prime}$ in $D(\xi)$ such that $\gamma_{\sigma^{\prime}(n)}\left(v_{n}^{\prime \prime}\right)=v$. If $D(\xi)$ is finite, after extracting another subsequence, we see that we are in the first case, but we supposed we were not. If $D(\xi)$ is infinite, we are in case (2) of the main theorem, and $D(\xi)$ is exactly the star of a vertex $v "$. If $v$ is in the orbit of the vertex stabilized by the group $A$, again, necessarily $\gamma_{\sigma^{\prime}(n)}\left(v^{\prime \prime}\right)=v$. If $v$ is not in this orbit, $\gamma_{\sigma^{\prime}(n)}^{-1} v$ ranges over infinitely many neighbours of $v$ ", therefore $\gamma_{\sigma^{\prime}(n)}^{-1} \partial\left(\Gamma_{v}\right)$ converges to the unique point of $\partial\left(\Gamma_{v^{\prime \prime}}\right)$ which we call $\zeta^{\prime}$. Therefore, the convergence is locally uniform away from $\zeta^{\prime}$, what we wanted to prove.

As an immediate corollary of the two previous lemmas, we have:

Corollary 3.3 With the previous notations, the group $\Gamma$ is a convergence group on $M$ (cf Definition 1.1).

Lemma 3.4 Every point in $\partial T \subset M$ is a conical limit point for $\Gamma$ in $M$.

Let $\eta \in \partial T$. Let $v_{0}$ a vertex in $T$ with a sequence $\left(\gamma_{n}\right)_{n \in \mathbb{N}}$ of elements of $\Gamma$ such that $\gamma_{n} v_{0}$ lies on the ray $\left[v_{0}, \eta\right)$, converging to $\eta$.

By Lemma 3.1, after possible extraction of subsequence, there is a point $\xi^{+} \in$ $M$, and for all $\xi \in M$, except possibly one in $\partial T$, we have $\gamma_{n}^{-1} \zeta \rightarrow \xi^{+}$. Note that, in particular, we have $\gamma_{n}^{-1} \partial\left(\Gamma_{v_{0}}\right) \rightarrow \xi^{+}$. By multiplying each $\gamma_{n}$ on the right by elements of $\Gamma_{v_{0}}$, we can assume that $\xi^{+}$is not in $\partial\left(\Gamma_{v_{0}}\right)$, and we still have $\gamma_{n} v_{0}$ lying on the ray $\left[v_{0}, \eta\right)$, converging to $\eta$.

Now it is enough to show that $\gamma_{n}^{-1} \cdot \eta$ does not converge to $\xi^{+}$. But $v_{0}$ is always in the ray $\left[\gamma_{n}^{-1} v_{0}, \gamma_{n}^{-1} \eta\right)$. Therefore, if $\gamma_{n}^{-1} \eta \rightarrow \xi^{+}$, this implies that $\xi^{+}$is in $\partial\left(\Gamma_{v_{0}}\right)$, which is contrary to our choice of $\left(\gamma_{n}\right)_{n \in \mathbb{N}}$.

Lemma 3.5 Every point in $\Omega / \sim$ which is image by $\pi$ of a conical limit point in a vertex stabilizer's boundary, is a conical limit point for $\Gamma$.

Such a point is in $\partial\left(\Gamma_{v}\right)$ for some vertex $v$, and it is a conical limit point in $\partial\left(\Gamma_{v}\right)$ for $\Gamma_{v}$. Therefore it is a conical limit point in $M$ for $\Gamma_{v}$ (see the remark (i) in section 1), hence for $\Gamma$. 
Lemma 3.6 Every point in $\Omega / \sim$ which is image by $\pi$ of a bounded parabolic point in a vertex stabilizer's boundary, is a bounded parabolic point for $\Gamma$. The maximal parabolic subgroup associated is the image in $\Gamma$ of a parabolic subgroup of a vertex group.

Let $\xi$ be the image by $\pi$ of a bounded parabolic point in a vertex stabilizer's boundary, let $D(\xi)$ be its domain, and $v_{1}, \ldots, v_{n}$ the (finite, by Proposition 2.2) list of vertices in $D(\xi)$ modulo the action of $\operatorname{Stab}(D(\xi))$, with stabilizers $\Gamma_{v_{i}}$. Let $P$ be the stabilizer of $\xi$. It stabilizes also $D(\xi)$, which is a bounded subtree of $T$. By the Serre fixed-point theorem, it fixes a point in $D(\xi)$, which can be chosen to be a vertex, since the action is without inversion. Therefore, $P$ is a maximal parabolic subgroup of a vertex stabilizer, and the second assertion of the lemma is true. For each $i \leq n$ the corresponding maximal parabolic subgroup $P_{i}$ of $\Gamma_{v_{i}}$ is a subgroup of $P$, because it fixes $\xi$. But for each $i \leq n$, $P_{i}$ is bounded parabolic in $\Gamma_{v_{i}}$, and acts properly discontinuously co-compactly on $\partial\left(\Gamma_{v_{i}}\right) \backslash\{\xi\}$.

For each index $i \leq n$, we choose $K_{i} \subset \partial\left(\Gamma_{v_{i}}\right) \backslash\{\xi\}$, a compact fundamental domain of this action. We consider also $\mathcal{E}_{i}$ the set of edges starting at $v_{i}$ whose boundary intersects $K_{i}$ and does not contain $\xi$. Let $e$ be an edge with only one vertex in $D(\xi)$, and $v_{i}$ be this vertex. As $K_{i}$ is a fundamental domain for the action of $P_{i}$ on $\partial\left(\Gamma_{v_{i}}\right) \backslash\{\xi\}$, there exists $p \in P_{i}$ such that $\partial\left(\Gamma_{e}\right) \cap p K_{i} \neq \emptyset$. Therefore, the set of edges $\bigcup_{i \leq n} P \mathcal{E}_{i}$ contains every edge with one and only one vertex in $D(\xi)$.

For each $i \leq n$, let $\mathcal{V}_{i}$ be the set of vertices $w$ of the tree $T$ such that the first edge of $\left[v_{i}, w\right]$ is in $\mathcal{E}_{i}$, and let $\overline{\mathcal{V}_{i}}$ be its closure in $T \cup \partial T$. Let $K_{i}^{\prime}$ be the subset of $M$ consisting of the points whose domain is included in $\overline{\mathcal{V}_{i}}$. As a sequence of points in the boundaries of the stabilizers of distinct edges in $\mathcal{E}_{i}$ has only accumulation points in $K_{i}$, the set $K_{i}^{\prime \prime}=K_{i} \cup K_{i}^{\prime}$ is compact. Hence $\bigcup_{i \leq n} K_{i}^{\prime \prime}$ is a compact space not containing $\xi$, and because $\bigcup_{i \leq n} P \mathcal{E}_{i}$ contains every edge with one and only one vertex in $D(\xi)$, the union of the translates of $\bigcup_{i \leq n} K_{i}^{\prime \prime}$ by $P$ is $M \backslash \xi$. Therefore, $P$ acts properly discontinuously co-compactly on $M \backslash \xi$.

We can summarize the results of this section:

Theorem 3.7 (Dynamic of $\Gamma$ on $M$ )

Under the conditions of Theorem 0.1, and with the previous notations, the group $\Gamma$ is a geometrically finite convergence group on $M$. 
The bounded parabolic points are the images by $\pi$ of bounded parabolic points, and their stabilizers are the images, and their conjugates, of maximal parabolic groups in vertex groups.

We are now able to prove our main theorem.

Proof of Theorem 0.1 The two first cases are direct consequences of Theorem 3.7 and of Theorem 1.5. The maximal parabolic subgroups are given by Lemma 3.6.

Cases (3) and $\left(3^{\prime}\right)$ can be deduced as follows. Let $\Gamma=G_{1} *_{P} G_{2}$, where $P$ is maximal parabolic in $G_{1}$ and parabolic in $G_{2}$. If $\widetilde{P}$ is the maximal parabolic subgroup of $G_{2}$ containing $P$, one has $\Gamma=\left(G_{1} *_{P} \widetilde{P}\right) *_{\widetilde{P}} G_{2}$. One can apply successively the second and the first case of the theorem to get the relative hyperbolicity of $\Gamma$. For the last case, If $\Gamma=G *_{P}$, then one can write $\Gamma=\left(G *_{P} P^{\prime}\right) *_{P^{\prime}}$, where $P^{\prime}$ is as in the statement, and apply consecutively the second and first case of the theorem. The acylindricity or the last HNNextension is given by the fact that the images of $P^{\prime}$ in the group $\left(G *_{P} P^{\prime}\right)$, are maximal parabolic subgroups not in the same conjugacy class.

\section{Relatively Hyperbolic Groups and Limit Groups}

In our combination theorem, the construction of the boundary helps us to get more information. For instance, we get an independent proof, and an extension to the relative case, of a theorem of I Kapovich [18] for hyperbolic groups.

Corollary 4.1 If $\Gamma$ is in Case (1) of Theorem 0.1, the vertex groups embed as fully quasi-convex subgroups in $\Gamma$.

The limit set of the stabilizer of a vertex $v$ is indeed $\partial\left(\Gamma_{v}\right)$. As domains are finite (Proposition 2.2 and its remark), a point in $M$ belongs to finitely many translates of $\partial\left(\Gamma_{v}\right)$.

Finally, we study limit groups, introduced by Sela in [24], in his solution of the Tarski problem, as a way to understand the structure of the solutions of an equation in a free group. We give the definition of limit groups; it involves a Gromov-Hausdorff limit. Here, we do not discuss the existence of such a limit, but we advise the reader to refer to Sela's original paper. 
Definition 4.2 (Limit groups, [24])

Let $G$ be a finitely generated group, with a finite generating family $S$, and $\gamma=\left(\gamma_{1} \ldots \gamma_{k}\right)$ a prescribed set of $k$ elements in $G$. Let $F$ be a free group of rank $k$ with a fixed basis $a=\left(a_{1} \ldots a_{k}\right)$, and let $X$ be its associated Cayley graph (it is a tree). Let $H(G, F ; \gamma, a)$ be the set of all the homomorphisms of $G$ in $F$ sending $\gamma_{i}$ on $a_{i}$. Each element of $H(G, F ; \gamma, a)$ naturally defines an action of $G$ on $X$. Let $\left(h_{n}\right)_{n \in \mathbb{N}}$ be a sequence of homomorphisms in distinct conjugacy classes, and let us rescale $X$ by a constant $\mu_{n}=\min _{f \in F} \max _{g \in S}\left(d_{X}\left(i d, f h_{n}(g) f^{-1}\right)\right)$ to get the pointed tree $\left(X_{n}, x_{n}\right)$, whose base point $x_{n}$ is the image of a base point in $X$. There is a subsequence such that $\left(X_{\sigma(n)}, x_{\sigma(n)}\right)$ converges in the sense of Gromov-Hausdorff, and let $\left(X_{\infty}, y\right)$ be the real tree that is the Gromov-Hausdorff limit, on which the group $G$ acts. Let $K_{\infty}$ be the kernel of this action (the elements of $G$ fixing every point in $\left.X_{\infty}\right)$. We say that the quotient $L_{\infty}=G / K_{\infty}$ is a limit group.

An important property of limit groups is an accessibility theorem, proven by Sela. Every limit group has a height: limit groups of height 0 are the finitely generated torsion-free abelian groups, and every limit groups of height $n>0$ can be constructed by finitely many free products, HNN-extensions or amalgamations of limit groups of height at most $(n-1)$, over cyclic groups (this is a consequence of Theorem 4.1 in [24]). We need only this fact, and the fact that every abelian subgroup of a limit group is contained in a unique maximal abelian subgroup (Lemma 1.4 in [24]). Limit groups are known to enjoy many more powerful properties, therefore, one can hope that a similar argument than ours would work for a wider class of groups. We now establish properties of acylindricity, which can be also found in [24].

Lemma 4.3 One can choose the accessibility splitings of a limit group to be acylindrical. Moreover, the edge group of a spliting involved is a maximal abelian subgroup, and malnormal, in at least one of the adjacent vertex groups.

If an amalgamation $A *_{Z} B$ is involved in the accessibility, then the subgroup $Z$ is maximal abelian in either $A$ or $B$, since it is a property of limit groups that it has to belong to a unique maximal abelian subgroup of the ambiant group. In particular $Z$ is malnormal in either $A$ or $B$, since if it was not, a proper subgroup would be in two distinct maximal abelian subgroups. Hence the amalgamation is 3 -acylindrical.

If an HNN-extension $A *_{Z}$ is involved, then, let $Z_{1}$ and $Z_{2}$ be the two images of $Z$ in $A$ in the extension, and let $t$ be a generator of the loop of the graph of 
group. If $Z_{1}$ is not maximal abelian, let $a_{1}$ be an element, not in $Z_{1}$, and in the unique maximal abelian subgroup of $A$ containing $Z_{1}$. If one conjugates $a_{1}$ by $t$, one gets an element of $A *_{Z}$ not in $A$, that commutes with $Z_{2}$. Then $Z_{2}$ is maximal abelian in $A$, as if it was not, it would not be in a unique maximal abelian subgroup of $A * Z$. Therefore, as in the case of amalgamations, we see that either $Z_{1}$ or $Z_{2}$ has to be maximal abelian, and therefore malnormal. Now, unless $Z_{1}=Z_{2}$, we see that they cannot intersect non-trivially, because they would span a larger maximal abelian subgroup, contradicting what we just proved. Therefore, the HNN-extension is 2-acylindrical. Finally, if $Z_{1}=Z_{2}$, note that $A *_{Z}=\left(A *_{Z} Z\right) *_{Z}=A *_{Z}\left(Z *_{Z}\right)=A *_{Z} Z^{2}$, which is a previous case.

From this accessibility, Sela deduces that limit groups are exactly the finitely generated $\omega$-residually free groups: these are the groups such that, for every finite family of non-trivial elements, there exists a morphism in a free group that is non trivial on each of these elements.

We will need the general fact:

Lemma 4.4 Let $(G, \mathcal{G})$ be a relatively hyperbolic group, and let $Z$ be a non parabolic infinite cyclic subgroup of $G$ which is its own normalizer. Let $\mathcal{Z}$ be the set of conjugates of $G$. Then $(G,(\mathcal{G} \cup \mathcal{Z}))$ is a relatively hyperbolic group.

To see this, note that the space $M$ obtained from $\partial(G)$ by identifying for each conjugate of $Z$, the two points of its limit set to a point, is Hausdorff because the sequence of the diameters of the preimages in $\partial(G)$ of any sequence of points in $M$ tends to zero (this is a consequence of Proposition 1.8, for instance). Therefore, $M$ is a compact metrisable space, on which the group $G$ acts as a convergence group. The images in $M$ of bounded parabolic points of $\partial(G)$ are still bounded parabolic points, with same stabilizers. If $\xi \in M$ is the image of a conical limit point, not in the limit set of some conjugate of $Z$, there is a sequence $\left(g_{n}\right)$ in $G$, and $a$ and $b$ distinct points of $\partial(G)$ such that $g_{n} \xi \rightarrow a$ and $g_{n} \zeta \rightarrow b$ for all other $\zeta$. If $a$ and $b$ map to the same point in $M$, then they are in the limit set of a same conjugate $Z^{\prime}$ of $Z$. We assumed that $\xi$ is not in the limit set of $Z^{\prime}$. Then by multiplying the $g_{n}$ by sufficiently large elements $z_{n}$ of $Z^{\prime}$ we would get that $z_{n} g_{n} \xi \rightarrow a, z_{n} g_{n} b \rightarrow b$ and for a sequence of points $a_{n}$ tending to $a$ more slowly than $g_{n} \xi, z_{n} g_{n} a_{n} \rightarrow c$ a point in a fundamental domain of $Z^{\prime}$ acting on $\partial(B) \backslash \Lambda Z^{\prime}$. In particular, this violates the convergence property. Therefore the images in $M$ of $a$ and $b$ are distinct. Hence, the sequence $g_{n}$ and the images of $a$ and $b$ in $M$ show that $\xi$ is a conical limit point. 
If $\xi \in M$ is the image of the limit set of $Z$ (which consists of two loxodromic fixed points), then its stabilizer is the normalizer of $Z$, that is $Z$ itself. As the cyclic group $Z$ acts co-compactly on the complement of its limit set in $\partial(G)$, (this is a consequence of the fact that $Z$ acts as a convergence group on $\partial G$ fixing the two points in its limit set), we see that $\xi$ is a bounded parabolic point in $M$. Similar fact is true for every conjugate of $Z$. All this together proves the relative hyperbolicity, by Theorem 1.5.

Theorem 4.5 [Theorem 0.3]

Every limit group is hyperbolic relative to the family of its maximal non-cyclic abelian subgroups.

We argue by induction on the height. It is obvious for groups of height 0 . Consider an HNN extension $A *_{Z}$ or an amalgamation $A *_{Z} B$, with $A$ and $B$ of height at most $(n-1), Z$ cyclic. If $Z$ is trivial or has cyclic centralizer in the amalgamation, both of its images in the vertex group(s) are fully quasi-convex, because it has trivial intersection with every non-cyclic abelian subgroup. Hence, the first case of the combination theorem gives the result.

Assume now that $A$ contains a maximal non-cyclic abelian subgroup containing $Z$. We consider the case of an amalgamation $A *_{Z} B$, the case of an HNN-extension being similar. Let $\left\{P_{i}\right\}$ be the set of maximal parabolic subgroups of $B$; each $P_{i}$ is a non-cyclic abelian group. From the discussion on the accessibility, we know that the group $Z$ is a maximal cyclic subgroup of $B$ not intersecting any of the $P_{i}$, and is malnormal in $B$. In particular, it is fully quasi-convex in $B$, and we note $Z_{i}$ the set of conjugates of $Z$. From Lemma 4.4, we have that $B$ is hyperbolic relative to $\left\{P_{i}\right\} \cup\left\{Z_{i}\right\}$.

We can apply the third case of Theorem 0.1 , this gives that $A *_{Z} B$ is hyperbolic relative to its maximal non-cyclic abelian subgroups, and this ends the proof for amalgamations.

The proof is similar in the case of an HNN-extension, using the case $\left(3^{\prime}\right)$ of the combination theorem, instead of the third case.

The next proposition was suggested by G Swarup (see also [28]). It was already known that every finitely generated subgroup of a limit group is itself a limit group (it is obvious if one thinks of $\omega$-residually free groups).

Proposition 4.6 (Local quasi-convexity)

Every finitely generated subgroup of a limit group is quasi-convex (in the sense of Definition 1.6). 
Again, we argue by induction on the height of limit groups.

The result is classical for free groups, surface groups, and abelian groups. Assume now that the property is true for $A$ and $B$, and consider $\Gamma=A *_{Z} B$, and $H$ a finitely generated subgroup of $\Gamma . H$ acts on the Serre tree $T$ of the amalgamation. In particular it acts on its minimal invariant subtree. As a consequence of the fact that $H$ is finitely generated, the quotient of this tree is finite. Moreover, as the edge groups are all cyclic or trivial, $H$ intersects each stabilizer of vertex along a finitely generated subgroup. Therefore, one gets a spliting of $H$ as a finite graph of groups, the vertex groups of which are finitely generated subgroups of the conjugates of $A$ and $B$, and with cyclic or trivial edge groups. As they are finitely generated, and by the induction assumption, the vertex groups are quasi-convex in the conjugates of $A$ and $B$, and their boundaries equivariantly embed in the translates of $\partial A$ and $\partial B$. We can apply our combination theorem on this acylindrical graph of groups, and as the Serre tree of the splitting of $H$ embeds in the Serre tree of the splitting of $\Gamma$, its boundary equivariantly embeds in $\partial T$. Thus, $H$ is a geometrically finite group on its limit set in the boundary of $\Gamma$, hence it is quasi-convex in $\Gamma$.

The Theorem 4.7 (Howson property for limit groups) was motivated by a discussion with G Swarup. To prove it, we first prove the Proposition 4.8, inspired by some results in [27]: we study the intersection of (not necessarly fully) quasiconvex subgroups.

This study completes the work of I Kapovich, who proved the Howson property for limit groups without any non-cyclic abelian subgroup (see [19] and [20]).

Theorem 4.7 Limit groups have the Howson property: the intersection of two finitely generated subgroups is finitely generated.

We postpone the proof, because we need the following:

Proposition 4.8 (Intersection of quasi-convex subgroup)

Let $\Gamma$ be a relatively hyperbolic group, with only abelian parabolic subgroups. Let $Q_{1}$ and $Q_{2}$ be two quasi-convex subgroups. Then $Q_{1} \cap Q_{2}$ is quasi-convex. Moreover, $\Lambda\left(Q_{1} \cap Q_{2}\right)$ differs from $\Lambda\left(Q_{1}\right) \cap \Lambda\left(Q_{2}\right)$ only by isolated points.

Let $Q_{1}$ and $Q_{2}$ be two quasi-convex subgroups of $\Gamma$ and $Q=Q_{1} \cap Q_{2}$. The limit sets satisfy $\Lambda(Q) \subset \Lambda\left(Q_{1}\right) \cap \Lambda\left(Q_{2}\right)$, and the action of $Q$ on $\Lambda(Q)$ is of convergence. As in Proposition 1.10, the conical limit points in $\Lambda(Q)$ are 
exactly the conical limit points in $\Lambda\left(Q_{1}\right)$ and in $\Lambda\left(Q_{2}\right)$. We want to prove that each remaining point in $\Lambda(Q)$ is a bounded parabolic point. Those points are among the parabolic points in both $\Lambda\left(Q_{1}\right)$ and $\Lambda\left(Q_{2}\right)$, but it may happen that a parabolic point for $Q_{1}$ and $Q_{2}$ is not in $\Lambda(Q)$.

However, it is enough to prove that, for all $p$, parabolic point for $Q_{1}$ and $Q_{2}$, then the quotient $\operatorname{Stab}_{Q}(p) \backslash\left(\Lambda\left(Q_{1}\right) \cap \Lambda\left(Q_{2}\right) \backslash\{p\}\right)$ is compact. Indeed, if we manage to do so, we would have proven that $\Lambda(Q)$ differs from $\Lambda\left(Q_{1}\right) \cap \Lambda\left(Q_{2}\right)$ only by isolated points: the parabolic points for $Q_{1}$ and $Q_{2}$ whose stabilizer in $Q$ is finite. Such a point $p$ is isolated, because the statement above implies that $\left(\Lambda\left(Q_{1}\right) \cap \Lambda\left(Q_{2}\right) \backslash\{p\}\right)$ is compact. Therefore, Proposition 4.8 follows from the general lemma:

Lemma 4.9 Let $G$ be a finitely generated abelian group, acting properly discontinuously on a space $E$. Assume that $G$ contains two subgroups, $A$ and $B$, such that $G=A B$. If $A$ acts on $X \subset E$ with compact quotient, and if $B$ acts similarly on $Y \subset E$, then $A \cap B$ acts properly discontinuously on $X \cap Y$, with compact quotient.

The only thing that needs to be checked is that the quotient is compact. Let $K_{A} \subset X$ be a compact fundamental domain for $A$ in $X$, and $K_{B}$ similarly for $B$ in $Y$. For all $a \in A$ such that $a K_{A} \cap Y \neq \emptyset$, there exists $b \in B$ such that $a K_{A} \cap b K_{B} \neq \emptyset$. As $K_{A}$ and $K_{B}$ are compact, and since the action of $(A+B)$ is properly discontinuous, there are finitely many possible values in $G$ for $a^{-1} b$, with $a$ and $d$ satisfying $a K_{A} \cap b K_{B} \neq \emptyset$. Therefore, for all such $a$ and $b$, there exists a word $w$ written with an alphabet of generators of $G$ consisting of generators of $A$ and generators of $B$, of length bounded by a number $N$ neither depending on $a$ nor on $b$, such that, in $G, w=a^{-1} b$. Using abelianity of the group $G$, we can gather the letters in $w$ in order to get a new word of same length, concatenation of two smaller ones: $w^{\prime}=w_{A} w_{B}$ with $w_{A} \in A$ and $w_{B} \in B$, and still, in $G, w^{\prime}=a^{-1} b$. Now we see that $a w_{A}=b\left(w_{B}\right)^{-1}$, and therefore $a w_{A} \in(A \cap B)$. If we set $K=\left(\bigcup_{\left|w_{A}\right| \leq N} w_{A} K_{A}\right) \cap Y$, which is compact, we have just shown that $(A \cap B) K$ covers $X \cap Y$. That is that we have proven the lemma.

Now we can prove the Howson property.

Proof of Theorem 4.7 Two finitely generated subgroups of a limit group are quasiconvex by Proposition 4.6, therefore, by Proposition 4.8, the intersection is also quasiconvex. In particular, by remark (ii) in section 1, it is finitely generated. 
We finally give an application of [9]. Following Bestvina [2], we say that a $\mathcal{Z}-$ structure (if it exists) on a group is a minimal (in the sense of $\mathcal{Z}$-sets) aspherical equivariant, finite dimensional (for the topological dimension) compactification of a universal cover of a finite classifying space for the group, $E \Gamma \cup \partial(E \Gamma)$, such that the convergence of a sequence $\left(\gamma_{n} p\right)_{n}$ to a point of the boundary $\partial(E \Gamma)$ does not depend on the choice of the point $p$ in $E \Gamma$ (see [2], [9]).

Theorem 4.10 (Topological compactification)

Any limit group admits a $\mathcal{Z}$-structure in the sense of [2].

The maximal parabolic subgroups are isomorphic to some $\mathbb{Z}^{d}$, and therefore admits a finite classifying space with a $\mathcal{Z}$-structure (the sphere that comes from the $C A T(0)$ structure). As limit groups are torsion free, (Lemma 1.3 in [24]), the main theorem of [9] can be applied to give the result.

We emphasize that this topological boundary needs not to be the one constructed above: if the group contains $\mathbb{Z}^{d}$, the topological boundary contains a sphere of dimension $d-1$.

\section{References}

[1] A Beardon, B Maskit, Limit points of Kleinian groups, and finite sided fundamental polyhedra, Acta Math. 132 (1974) 1-12

[2] M Bestvina, Local Homology Properties of Boundaries of Groups, Michigan Math. J. 43 (1996) 123-139

[3] M Bestvina, M Feighn, A Combination Theorem for Negatively Curved Groups, J. Differential Geom. 35 (1992) 85-101

[4] M Bestvina, M Feighn, Addendum and correction to 'A Combination Theorem for Negatively Curved Groups', J.Differential Geom. 43 (1996) 783-788

[5] B H Bowditch, Geometrical Finiteness With Variable Negative Curvature, Duke Math. J. 77 (1995) 229-274

[6] B H Bowditch, Convergence Groups and Configuration Spaces, from: "Geometric group theory down under (Canberra, 1996)" 23-54

[7] B H Bowditch, A Topological Characterisation of Hyperbolic Groups, J. Amer. Math. Soc. 11 (1998) 643-667

[8] B H Bowditch, Relatively Hyperbolic Groups, preprint Southampton (1999)

[9] F Dahmani, Classifying Spaces and Boundaries for Relatively Hyperbolic Groups, Proc. London Math. Soc. 86 (2003) 666-684 
[10] F Dahmani, Les groupes relativement hyperboliques et leurs bords, $\mathrm{PhD}$ thesis, Strasbourg (2003)

[11] B Farb, Relatively Hyperbolic Groups, Geom. Funct. Anal. 8 (1998) 810-840

[12] F Gehring, G Martin, Discrete Quasi-conformal Groups, Proc. London Math. Soc. 55 (1987) 331-358

[13] R Gitik, On the Combination Theorem for Negatively Curved Groups, Internat. J. Algebra Comput. 7 (1997) 267-276

[14] M Gromov, Hyperbolic Groups, from: "Essays in group theory", (S Gersten, editor) MSRI (1987) 75-263

[15] M Gromov, Asymptotic Invariants of Infinite Groups, from: "Geometric group theory, Vol. 2", London Math. Soc. Lecture Note Ser. 182 (1993)

[16] W Hurewicz, H Wallman, Dimension theory, Princeton university press (1946)

[17] I Kapovich, Quasiconvexity and Amalgams, Internat. J. Algebra Comput. 7 (1997) 771-811

[18] I Kapovich, Quasi Convexity and the Combination Theorem, Internat. J Algebra Comput. 11 (2001) 185-216

[19] I Kapovich, Subgroup Properties of Fully Residually Free Groups, Trans. Amer. Math. Soc 354 (2001) 335-362

[20] I Kapovich, Erratum to 'Subgroup properties of fully residually free groups', Trans. Amer. Math. Soc. 355 (2003) 1295-1296

[21] O Kharlampovich, A Myasnikov, Hyperbolic Groups and Free Constructions, Trans. Amer. Math. Soc 350 (1998) 571-613

[22] O Kharlampovich, A Myasnikov, Irreducible Affine Varieties over a Free Group. I and II, J. Algebra 200 (1998) 472-570

[23] Z Sela, Acylindrical Accessibility for Groups, Invent. math. 129 (1997) 527-565

[24] Z Sela, Diophantine Geometry over Groups I: Makanin-Razborov diagrams, IHES Publ. Math. 93 (2001) 31-105

[25] Z Sela, Diophantine Geometry over Groups: A list of Research Problems, available at http://www.ma.huji.ac.il/ zlil/

[26] H Short, Quasiconvexity and a Theorem of Howson's, from "Group Theory From a Geometrical Viewpoint (Trieste, 1990)", World Sci. Publishing, River Edge, NJ (1991) 168-176

[27] P Susskind, G Swarup, Limit Sets of Geometrically Finite Hyperbolic Groups, Amer. J. Math. 114 (1992) 233-250

[28] G Swarup, Proof of a Weak Hyperbolization Theorem, Q. J. Math. 51 (2000) 529-533

[29] A Szczepański, Relatively Hyperbolic Groups, Michigan Math. J. 45 (1998) 611-618 
[30] P Tukia, Generalizations of Fuchsian and Kleinian groups, First European Congress of Mathematics, Vol. II (Paris, 1992) 447-461

[31] P Tukia, Conical Limit Points and Uniform Convergence Groups, J. Reine Angew. Math. 501 (1998) 71-98

[32] A Yaman, A Topological Characterisation of Relatively Hyperbolic Groups, to appear in J. Reine Angew. Math. 